\title{
Determinantes da interrupção precoce do aleitamento materno: uma revisão narrativa
}

\author{
Determinants of early cessation of breastfeeding: a narrative review
}

Determinantes del cese temprano de la lactancia materna: una revisión narrativa

Bruna Abaliac Azevedo ${ }^{1 *}$, Rita de Cássia Natividade Ataíde.

\section{RESUMO}

Objetivo: Analisar na literatura científica sobre as motivações e os principais motivos que podem gerar o desmame precoce. Revisão bibliográfica: Amamentar é um processo que envolve interação entre mãe e filho, trazendo uma série de consequências benéficas, não apenas para o estado nutricional da criança, como para seu desenvolvimento cognitivo-emocional. Além disso, atua na defesa contra infecções, impactando na saúde do lactente em longo prazo. E ainda promove a saúde física e psíquica da mãe. Apesar de todas as comprovações científicas confirmarem a superioridade da amamentação sobre outras formas de nutrir a criança, do empenho de inúmeros organismos nacionais e internacionais e das campanhas promovidas, a prevalência do aleitamento materno no Brasil, em especial da amamentação exclusiva, está distante da recomendada. Considerações finais: É fundamental que os profissionais de saúde sejam capacitados para orientação e empoderamento das mães, oferecendo um suporte ativo, inclusive emocional, fornecendo referências precisas e desmitificando alguns tabus. Igualmente importante são as políticas de saúde voltadas à orientação sobre temas que geram dúvidas e incertezas durante a gravidez, incluindo a importância da amamentação exclusiva até os seis meses de vida do bebê e demonstrando os malefícios do desmame precoce.

Palavras-chave: Aleitamento materno, Desmame precoce, Determinantes, Políticas públicas.

\begin{abstract}
Objective: Analyze the scientific literature on the motivations and main reasons that can generate early weaning. Bibliographic review: Breastfeeding is a process that involves interaction between mother and child, bringing a series of beneficial consequences, not only for the child's nutritional status, but also for their cognitive-emotional development. In addition, it acts in defense against infections, impacting the infant's health in the long term. And it also promotes the mother's physical and mental health. Despite all the scientific evidence confirming the superiority of breastfeeding over other ways of nurturing the child, the commitment of numerous national and international organizations and the campaigns promoted, the prevalence of breastfeeding in Brazil, especially exclusive breastfeeding, is far from the recommended. Final considerations: It is essential that health professionals are trained to guide and empower mothers, offering active support, including emotional support, providing accurate references and demystifying some taboos. Equally important are health policies aimed at providing guidance on issues that generate doubts and uncertainties during pregnancy, including the importance of exclusive breastfeeding until the baby is six months old and demonstrating the harm of early weaning.
\end{abstract}

Keywords: Breastfeeding, Early weaning, Determinants, Public policies.

${ }^{1}$ Faculdade Santo Agostinho (FASA), Vitória da Conquista - BA. *E-mail: brunaabaliac@hotmail.com 


\section{RESUMEN}

Objetivo: Analizar la literatura científica sobre las motivaciones y principales motivos que pueden generar el destete precoz. Revisión bibliográfica: La lactancia materna es un proceso que involucra la interacción entre madre e hijo, trayendo una serie de consecuencias beneficiosas, no solo para el estado nutricional del niño, sino también para su desarrollo cognitivo-emocional. Además, actúa en defensa contra infecciones, impactando la salud del bebé a largo plazo. Y también promueve la salud física y mental de la madre. A pesar de todas las evidencias científicas que confirman la superioridad de la lactancia materna sobre otras formas de crianza del niño, el compromiso de numerosas organizaciones nacionales e internacionales y las campañas promovidas, la prevalencia de la lactancia materna en Brasil, especialmente la lactancia materna exclusiva, está lejos de la recomendada. Consideraciones finales: Es fundamental que los profesionales de la salud estén capacitados para orientar y empoderar a las madres, ofreciendo apoyo activo, incluido el apoyo emocional, brindando referencias precisas y desmitificando algunos tabúes. Igualmente importantes son las políticas de salud dirigidas a orientar sobre temas que generan dudas e incertidumbres durante el embarazo, incluida la importancia de la lactancia materna exclusiva hasta los seis meses de edad y demostrar los daños del destete precoz.

Palabras clave: Lactancia materna, Destete precoz, Determinantes, Políticas públicas.

\section{INTRODUÇÃO}

Amamentar é um processo que envolve interação entre mãe e filho, trazendo uma série de consequências benéficas não apenas para o estado nutricional da criança, como para o seu desenvolvimento cognitivoemocional. Além disso, atua na defesa contra infecções, impactando na saúde do lactente a longo prazo e ainda promove a saúde física e psíquica da mãe. A amamentação é um dos métodos que mais colabora para a prevenção de mortes infantis, conseguindo salvar mais de 800.000 vidas em menores de cinco anos de idade por ano, em todo o mundo, além de contribuir na diminuição da mortalidade neonatal (OLIVEIRA DS, et al., 2017).

Diversas instituições nacionais e internacionais, a exemplo do Ministério da Saúde (MS), Fundo das Nações Unidas para a Infância (UNICEF), a Organização Mundial de Saúde e a Organização Pan-Americana de Saúde (OMS/OPAS), recomendam a amamentação exclusiva até o sexto mês de vida do bebê. Estas entidades consideram o leite materno (LM) a principal estratégia para a nutrição da criança na primeira infância e uma das principais estratégias para a redução da mortalidade infantil. Isso é possível graças à composição do leite humano (LH) que contém lipídeos, proteínas, substâncias imunocompetentes (como imunoglobulina $A$ e enzimas), fatores tróficos ou moduladores de crescimento, além de ácidos graxos fundamentais no desenvolvimento do recém-nascido e muito mais (SILVA CM, et al., 2017).

Ademais, não há vantagens na introdução de alimentos complementares antes dos seis meses de vida, pois pode ocasionar prejuízos à saúde da criança sobre inúmeros aspectos. Dentre os riscos encontram-se a menor duração do aleitamento materno (AM), episódios frequentes de diarreia, hospitalizações por doença respiratória, desnutrição quando os alimentos inseridos são inferiores do ponto de vista nutricional, menor absorção de nutrientes do leite materno e menor efetividade da amamentação como método anticoncepcional. O abandono total ou parcial do aleitamento materno, com introdução de outros tipos de alimentos diferentes do LH antes da criança completar 180 dias de vida, é denominado desmame precoce (MONTESCHIO CAC, et al., 2015).

Diversos fatores competem para o desmame precoce, como adição de água e líquidos não nutritivos, desnecessários antes dos seis meses de vida; uso de chupeta e mamadeira no início da amamentação; falta de conhecimento das mulheres sobre os benefícios do aleitamento materno exclusivo (AME) até o sexto mês; falta da amamentação na primeira hora após o parto; parto cesáreo; estado de saúde físico e mental da mãe; depressão pós-parto; inserção precoce das puérperas no mercado de trabalho; baixa escolaridade; introdução de outros alimentos precocemente; situação socioeconômica e cultural desfavorável e falta de acompanhamento e orientação dos profissionais de saúde (FONSECA PCA, et al., 2017). 
Um estudo com 1.118 gestantes de alto risco que realizaram pré-natal em um centro especializado da rede do Sistema Único de Saúde (SUS) detectou que elas apresentavam altas taxas de intenção de amamentação exclusiva, sobretudo aquelas que possuíam escolaridade mais elevada, que tiveram outros filhos amamentados e contaram com apoio familiar. Os principais motivos apontados para a falta da intenção de amamentar de forma exclusiva foram os seguintes: uso de diversos tipos de medicamentos, convivência com o Vírus da Imunodeficiência Humana (HIV), trabalho fora de casa e falta de informações sobre o processo e o valor do aleitamento materno (MOIMAZ SAS, et al., 2014).

Além disso, as taxas de amamentação exclusiva em crianças menores de seis meses variaram entre $20 \%$ a 59\%. Quando analisados apenas recém-nascidos prematuros de baixo peso, ficaram entre $19,5 \%$ a $76 \%$ no âmbito hospitalar, caindo em mais de $50 \%$ no período compreendido entre a alta do bebê e o primeiro retorno ao ambulatório (FLORES TR, et al., 2017).

Apesar de todas as comprovações científicas da superioridade da amamentação sobre outras formas de nutrição, do empenho de inúmeros organismos nacionais e internacionais e das campanhas promovidas, a prevalência do aleitamento materno no Brasil, principalmente de forma exclusiva, está distante da recomendada, configurando-se num dos mais importantes problemas de saúde pública do país. Outro aspecto identificado como fundamental neste processo diz respeito à compreensão da mulher em sua dimensão subjetiva, perpassando pelo seu reconhecimento como protagonista na prática da amamentação (KALIL IR e AGUIAR AC, 2017).

Persiste, portanto, a necessidade de identificação de novos fatores associados ao desmame precoce, com suas desastrosas consequências, para aprimoramento do incentivo à prática do aleitamento materno, garantindo a sua duração. Na medida em que se conhece os reais motivos é possível fazer a prevenção, atuando de forma mais objetiva e eficiente (CIAMPO LAD e CIAMPO IRLD, 2018).

Assim, diante desses pressupostos e considerando a relevância do tema abordado, esse constructo possui como objetivo analisar na literatura científica sobre as motivações e os principais motivos que podem gerar o desmame precoce.

\section{REVISÃO BIBLIOGRÁFICA}

\section{Conceitos importantes sobre aleitamento materno}

A oferta do LH é um direito incontestável da mãe e seu filho, assim, todos os esforços devem ser empreendidos no sentido de promover, acompanhar e manter o AME até os seis meses e complementá-lo até os dois anos de idade. $O$ ato de amamentar é uma fonte de troca de calor, conforto e amor, sendo fundamental para os desenvolvimentos emocional e psíquico da criança. Com isso, os benefícios da amamentação são incontestáveis para o crescimento saudável da criança do ponto de vista biopsicossocial, assim como da saúde da mãe e, porque não dizer, da família e da sociedade, constituindo-se num ato fundamental para o ser humano. No entanto, fatores socioeconômicos e culturais podem favorecer 0 desmame precoce e o abandono da amamentação por sua falta de valorização (SANTOS FS, et al., 2015).

A introdução de alimentos complementares antes dos seis meses, salvo em alguns casos específicos, não trará vantagens para a criança, ao contrário, pode causar prejuízos para a saúde. As crianças que foram amamentadas de forma exclusiva até os seis meses tiveram menos infecções intestinais, hospitalizações por doenças respiratórias e não tiveram déficits de crescimento, quando foram comparadas com as que receberam uma alimentação complementar, em adição ao leite materno, dos três aos quatro meses de vida (CAMINHA MFC, et al., 2015).

Crianças amamentadas exclusivamente com LH têm menor probabilidade de desenvolver doenças crônicas não transmissíveis na infância, adolescência e até durante a vida adulta. Apesar das vantagens que o AM oferece, existem diversos fatores que contribuem para a diminuição desta prática em várias partes do mundo. No Brasil, um dos fatores mais frequentes é a inserção da mulher no mercado de trabalho, estimulando, assim, o desmame precoce (KALIL IR e AGUIAR AC, 2016). 
O desmame precoce pode ser influenciado por diversos fatores, sejam eles biológicos, histórico-culturais, econômico-sociais e psíquicos, sendo um método com muitas ideologias e determinantes que resultam de condições inconscientes e concretas de vida. Diversas patologias estão relacionadas ao desmame precoce nos lactentes. Uma delas é a ruptura do desenvolvimento motor-oral adequado, causando alterações na postura e na força dos órgãos fonoarticulatório (lábios, língua, mandíbula, maxila, bochechas, palato mole, palato duro, assoalho da boca, musculatura oral e arcadas dentárias), afetando as funções de mastigação, deglutição, respiração e articulação dos sons da fala. Outra possibilidade são as alterações nas funções de defesa orgânica, podendo levar à morte (SILVA CM, et al., 2017).

No Brasil têm sido observados alguns avanços nas ações do Programa de Atenção Básica do Ministério da Saúde, reorganizando as políticas públicas de efetivação e estímulo ao AM, resultando em sua melhoria. No entanto, a permanência do AME até os seis meses de vida ainda é considerado um desafio, assim como para diversos países, devido a diversas peculiaridades culturais, sociais, econômicas e geográficas (BINNS C, et al., 2016).

\section{Vantagens da lactação}

A amamentação é o ato mais natural que existe e possui inúmeros benefícios nutricionais, emocionais e econômicos favorecendo, consequentemente, que a criança tenha um adequado crescimento e desenvolvimento facial, mantendo uma proximidade corporal repleta de sentidos entre a mãe e o filho. É benéfico para ambos, além do efeito positivo para a sociedade (SIPSMA HL, et al., 2018).

Considera-se que a amamentação traz benefícios psicológicos para a dupla mãe-bebê, permitidos pelo contato contínuo entre os dois, olhos nos olhos, fortalecendo os laços afetivos. Com isso, aumenta a intimidade, a transferência de afeto, os sentimentos de proteção e de segurança na criança, além da autoconfiança e realização da mulher. Acredita-se também que exista uma possível relação entre aleitamento materno e melhor desenvolvimento cognitivo, apesar do mecanismo não ser plenamente conhecido (SANTOS FS, et al., 2015).

Além disso, existe a superioridade do LH sobre os leites de outras espécies, pois ele possui todos os nutrientes necessários para o crescimento e desenvolvimento ideais da criança pequena, além de ser melhor digerido, quando comparado ao leite de outros animais. As necessidades nutricionais da criança nos primeiros seis meses de vida conseguem ser suprida apenas pelo consumo do $\mathrm{LH}$, e este continua sendo uma importante fonte de nutrientes principalmente de proteínas, vitaminas e gorduras, no segundo ano de vida. (MINISTÉRIO DA SAÚDE, 2015).

O consumo de pequenas quantidades do leite de vaca nos primeiros dias de vida amplia o risco de alergia a este leite, e se for utilizado antes dos quatro meses de idade é considerado um determinante do Diabetes Mellitus tipo 1, podendo aumentar em $50 \%$ o risco do seu aparecimento. As crianças que são amamentadas adoecem com menor frequência e com isso necessitam de uma quantidade menor de atendimento médico, medicamentos e hospitalização, reduzindo, assim, os custos, os eventos estressantes e o absenteísmo dos pais ao trabalho. Com isso, quando a amamentação é realizada com êxito, tanto as mães como as crianças ficam mais felizes, repercutindo nas relações e na qualidade de vida destas famílias (FLORES TR, et al., 2017).

Durante a amamentação a criança faz esforço para retirar o leite da mama, importante para o correto desenvolvimento da cavidade oral, proporcionando uma boa conformação do palato duro, do alinhamento adequado dos dentes e de uma correta oclusão dentária. Com o uso de chupetas e mamadeiras, o palato da criança é impulsionado para cima, elevando o assoalho da cavidade nasal e diminuindo o espaço utilizado para a passagem do ar, prejudicando a respiração pelo nariz. Com isso, o desmame precoce também pode levar ao desenvolvimento motor-oral inadequado, causando alteração das funções de mastigação, deglutição e articulação dos sons da fala, além de má-oclusão dentária e respiração bucal (GERTOSIO C, et al., 2016).

O AM parece estar relacionado à uma adequada saúde física e emocional da mãe durante o período do puerpério, na lactação e em toda sua vida futura. As mulheres que amamentam buscam com menor frequência um atendimento médico devido à redução das doenças respiratórias, cardiocirculatórias e 
gastrointestinais, bem como dos sintomas associados a problemas emocionais; as mães relataram se sentirem mais tranquilas, menos estressadas e agressivas, bem-humoradas e mais estimuladas a socializar desde os primeiros dias pós-parto (VICTORA CG, et al., 2016).

Além disso, a sucção precoce da região areal-mamilar é um dos estímulos essenciais para a produção de ocitocina, responsável pela contração uterina, fazendo com que o útero retorne mais rapidamente ao seu tamanho natural e limitando a possibilidade de ocorrência de hemorragia pós-parto e anemia. A ocitocina em quantidades elevadas pode aumentar o limiar da dor, diminuindo o desconforto materno (JONAS W e WOODSIDE B, 2016).

Mulheres que não amamentam têm um risco elevado de depressão durante o período pós-parto. A amamentação parece agir em um mecanismo de regulação da secreção diurna de cortisol, mantendo a concentração estável deste hormônio, reduzindo o risco de depressão pós-parto (DIAS CC e FIGUEIREDO $B, 2015)$. Além disto, a esclerose múltipla afeta principalmente as mulheres que se encontram em períodos férteis. O risco de reincidência reduz consideravelmente no decorrer da gravidez e da amamentação exclusiva. A duração do AM por 15 meses ou mais foi relacionada a um menor risco de esclerose múltipla, quando comparada com AM por menos de 4 meses (LANGER-GOULD A, et al., 2017).

Durante a lactação, os hormônios estrogênio e progesterona são suprimidos, levando a um período de infertilidade (WIJDEN CVD e MANION C, 2015). Para tanto, a mulher não pode ter menstruado e deve permanecer com o AME sob demanda de, no mínimo, oito vezes no decorrer do dia. A amenorreia que acontece durante o período da amamentação pode ser explicada pela inibição da ação ovariana devido aos elevados níveis de prolactina, gerando inibição do hormônio gonadotrofina e suspenção da ovulação (CHOWDHURY R, et al., 2015). Quando ocorre o regresso dos ciclos menstruais, a possibilidade de acontecer a concepção é reduzida em 7,4\% para cada mês adicional de amamentação (LABBOK MH, 2015).

A amamentação exclusiva ou predominante irá prolongar o espaçamento entre gestações e partos, tendo $98 \%$ de proteção nos primeiros seis meses após o parto e $96 \%$ depois. Outra vantagem é a praticidade de amamentar, dispensa de despesas com substitutos do LH, maior probabilidade da mãe retornar ao seu peso anterior à gestação, risco diminuído de artrite reumatoide e osteoporose aos 65 anos. Mulheres que amamentaram tem risco reduzido de desenvolver a doença de Alzheimer provavelmente, devido ao efeito do estrogênio sobre os receptores cerebrais. Foi observado também um aumento da sensibilidade à insulina desencadeada pelo AM (SANTOS ZB, 2018).

Mulheres que amamentam por, no mínimo, 02 meses, apresentam menor risco de desenvolver câncer no epitélio ovariano. E se a duração for de 03 a 24 meses, irá proteger contra o câncer de mama. A motivação das mães é um fator importante, pois auxilia no trajeto entre o desejo de amamentar e a concretização. Mas boa parte das mulheres abandona esta prática e inicia precocemente alimentos complementares à dieta da criança (SANTOS GCP, et al., 2017).

\section{Principais motivos relacionados ao desmame precoce}

A duração do AM pode sofrer influência de diversos fatores, tais como: condições sociodemográficas, ambientais, biológicas, obstétricas e culturais, além dos fatores relacionados à assistência prestada pelos serviços de saúde. As mães que possuem uma melhor condição socioeconômica têm, via de regra, um nível educacional mais elevado, o que permite melhor acesso à informação sobre a importância da amamentação, contribuindo de forma significativa para a sua prática, quando comparadas às mães com renda familiar baixa e/ou ocupação informal (SILVA VA, et al., 2019).

Com a crescente participação da mulher no mercado de trabalho, houve um aumento do risco de oferecimento antecipado de alimentos diferentes do LM, principalmente o leite de vaca, fazendo com que 0 regime alimentar seja incompatível com as diretrizes da alimentação saudável nos dois primeiros anos de vida (MONTEIRO FR, et al., 2017).

Apesar da maior parte dos profissionais da área de saúde se manifestarem a favor do aleitamento materno, diversas mulheres se mostram descontentes com o tipo de apoio recebido por eles. Tal fato pode ocorrer em decorrência de divergências na compreensão do que significa o apoio à amamentação. As mulheres que 
estão amamentando desejam ter um suporte ativo, incluindo emocional, com informações precisas, para se sentirem seguras nesse momento, mas infelizmente o suporte ofertado pelos profissionais costuma ser mais passivo e reativo (MINISTÉRIO DA SAÚDE, 2015).

Assim, a fragilidade dos profissionais de saúde no manuseio das questões associadas com a amamentação pode representar outro obstáculo ao AME. Eles encontram dificuldades para resolver questões práticas relacionadas a orientação às mães, repercutindo direta e negativamente nas taxas de AME. Assim, o manejo dos transtornos relacionados ao aleitamento materno requer diversas habilidades técnicas e relacionais que têm por base uma boa comunicação com a puérpera. Escuta sensível, preparo para identificar adversidades da nutriz, capacidade de promover e apoiar o aleitamento e auxiliar na superação das dificuldades iniciais são algumas das competências que os profissionais de saúde precisam adquirir para estarem aptos a este tipo de trabalho (BAPTISTA SS, et al., 2015).

Além disso, existe uma complexidade do AME, pois provoca nas mulheres sentimentos ambíguos, como desejo, alegria e satisfação, como também tristeza e insatisfação, uma situação que vai além da fisiologia, pois tem relação com a conjuntura estabelecida no ambiente em que a mulher está inserida e do apoio recebido diante dos obstáculos vivenciados. As mulheres podem apresentar sentimentos negativos, como tristeza, impotência e frustração frente à interrupção do AME (PRADO CVC, et al., 2016).

Expressões como "leite fraco" e "leite pouco" estão associadas à aparência aguada do leite materno e a sua pequena quantidade produzida. $O$ choro do bebê possui um significado de fome não saciada e de inconformidade, mesmo depois da mamada, suscitando insegurança materna e questionamentos quanto à capacidade de atender às demandas nutricionais do filho. Estes mitos e tabus contribuem para a alimentação complementar antes do sexto mês de vida da criança, por meio de fórmulas e sucos, desencadeando o desmame precoce (LIMA SP, et al., 2019).

Além disto, as dificuldades mais relatadas nos 15 dias após o parto foram "leite fraco" e/ou "pouco leite" $(39,2 \%)$ e trauma mamilar $(39,2 \%)$ como fatores concorrentes para o desmame precoce. O mito do leite fraco é um fator cultural, pois sabe-se que a maioria das mulheres tem leite suficiente para satisfazer a criança (ROLLINS NC, et al., 2016). Esta percepção equivocada pode estar relacionada a incompreensão do imaginário feminino quanto à composição e fisiologia do leite materno, associando assim, o choro do bebê à carência de alimento, o que nem sempre é verdade. O choro e a agitação do bebê, a percepção de fome e a incapacidade de tranquilizá-lo fazem com que a mãe pense que ela tem pouco leite, levando à introdução de substitutos (ROLLINS NC, et al., 2016).

Outro fator relacionado à interrupção precoce do AME e do aleitamento misto complementado é a ausência da realização do pré-natal. No Brasil, a atenção ao pré-natal na última década foi considerada inadequada, necessitando melhorias significativas em diversos aspectos, pois a realização indevida ou a ausência das consultas do pré-natal está relacionada a elevados índices de morbimortalidades materna e infantil (NUNES JT, et al., 2016).

É fundamental a implementação de políticas de saúde incentivando o pré-natal, com atenção voltada às necessidades subjetivas de cada mulher, ao vínculo entre profissionais e gestantes e aos procedimentos técnicos preconizados no pré-natal. Estes incluem escuta e orientação sobre os variados temas que geram dúvidas e incertezas durante a gravidez, além de orientações sobre o parto, pós-parto e promoção da saúde das gestantes (DOMINGUES RMSM, et al., 2015).

Acredita-se que as mães com idades mais avançadas tenham uma experiência prévia maior, seja pelas gestações anteriores ou pelas relações anteriores com os serviços de saúde durante o pré-natal, parto e puerpério. Já as mães adolescentes e jovens com poucos filhos ou nulíparas têm mais insegurança sobre a gestação e a amamentação (SILVA VA, et al., 2019).

A duração do aleitamento materno é maior nas mulheres pobres quando comparadas às mulheres ricas, nos países de baixa e média rendas, enquanto que nos países ricos, ocorre o inverso. Isso demonstra que os padrões de amamentação estão auxiliando na redução das discrepâncias em saúde entre crianças ricas e pobres em países de renda baixa e média, as quais seriam ainda maiores na privação da amamentação (VICTORA CG, et al., 2016). 
O desmame precoce também varia de acordo com o sexo da criança, sendo maior no sexo masculino (introdução prematura do leite de vaca) do que no feminino (benefício do AME). Tal diferença está relacionada à crença de que os meninos são biologicamente "mais fortes" e devem receber o "leite mais forte" que, erroneamente na concepção de algumas mães, é o leite de vaca (SILVA VA, et al., 2019).

O sucesso do AM depende da habilidade de sucção da criança, de uma boa técnica durante a amamentação e da anatomia das mamas. As mulheres que possuem os mamilos planos ou invertidos, geralmente encontram uma adversidade maior durante 0 aleitamento materno, pois o bebê não consegue abocanhar o mamilo corretamente, dificultando a técnica adequada para amamentar. Escoriações, fissuras, vermelhidão e ingurgitamento das mamas também são fatores que facilitam o desmame precoce (BARBOSA GEF, et al., 2017). Outro fator que pode desestimular a amamentação é a erupção dos dentes antes do sexto mês de vida, gerando pega ou posicionamento inadequado da criança ao mamar, o que leva ao aparecimento de dor durante o aleitamento (AMARAL LJX, et al., 2015).

Além disso, a ampliação dos locais destinados ao ato de amamentar no ambiente de trabalho contribui de forma significativa para maior duração do AM. Medidas como esta consolida a estratégia de rede social de apoio à amamentação, principalmente para as mães que trabalham, pois elas são um grupo de risco maior para o oferecimento precoce de alimentos complementares (FERNANDES VMB, et al., 2018).

\section{CONSIDERAÇÕES FINAIS}

O aleitamento materno possui benefícios que garantem o estado nutricional da criança, com ganhos consideráveis que serão percebidos ao longo da vida, tanto do ponto de vista físico quanto do desenvolvimento cognitivo-emocional. Desta forma, é fundamental que os profissionais de saúde sejam capacitados no acompanhamento e orientação das mães, demonstrando a importância da amamentação para o filho e para elas próprias, se constituindo num suporte ativo, de apoio emocional e desmitificando alguns tabus. Igualmente importantes são as políticas de saúde voltadas para orientação no pré-natal sobre os temas que geram dúvidas e incertezas durante a gravidez, incluindo a relevância da amamentação exclusiva até os primeiros seis meses de vida do bebê e demonstrando os malefícios do desmame precoce.

\section{REFERÊNCIAS}

1. AMARAL LJX, et al. Fatores que influenciam na interrupção do aleitamento materno exclusivo em nutrizes. Revista Gaúcha de Enfermagem, 2015; 36: 127-34.

2. BAPTISTA SS, et al. Manejo clínico da amamentação: atuação do enfermeiro na unidade de terapia intensiva neonatal. Revista de Enfermagem, 2015; 5(1): 23-31.

3. BARBOSA GEF, et al. Dificuldades iniciais com a técnica da amamentação e fatores associados a problemas com a mama em puérperas. Revista Paulista de Pediatria, 2017; 35(3): 265-272.

4. BINNS C, et al. Os benefícios de longo prazo da amamentação para a saúde pública. Asia Pacific Journal of Public Health, 2016; 28(1): 7-14.

5. CAMINHA MFC, et al. Fatores de risco para a não amamentação: um estudo caso-controle. Revista Brasileira de Saúde Materno Infantil, 2015; 15(2): 193-199.

6. CHOWDHURY R, et al. Amamentação e resultados de saúde materna: uma revisão sistemática e meta-análise. Acta Pediatria, 2015; 104(467): 96-113.

7. CIAMPO LAD, CIAMPO IRLD. Aleitamento materno e seus benefícios para a saúde da mulher. Revista Brasileira de Ginecologia e Obstetrícia, 2018; 40(6): 354-359.

8. DIAS CC, FIGUEIREDO B. Amamentação e depressão: uma revisão sistemática da literatura. The Journal of Affective Disorders, 2015; (171): 142-154.

9. DOMINGUES RMSM, et al. Adequação da assistência pré-natal segundo as características maternas no Brasil. Revista Panamericana Salud Pública, 2015; 37(3): 140- 7.

10. FERNANDES VMB, et al. Condutas de gestores relacionadas ao apoio ao aleitamento materno nos locais de trabalho. Texto \& Contexto-Enfermagem, 2018; 27(3): e2560016.

11. FLORES TR, et al. Consumo de leite materno e fatores associados em crianças menores de dois anos: Pesquisa Nacional de Saúde, 2013. Caderno de Saúde Pública, 2017; 33(11): 1-15.

12. FONSECA PCA, et al. Determinantes da velocidade média de crescimento de crianças até seis meses de vida: um estudo de coorte. Ciência e Saúde coletiva, 2017; 22(8): 2713-2726. 
13. GERTOSIO C, et al. Amamentação e sua gama de benefícios. Minerva Pediatria, 2016; 68(3): $201-212$.

14. JONAS W, WOODSIDE B. Mecanismos fisiológicos, fatores comportamentais e psicológicos que influenciam a transferência de leite das mães para seus filhos. Hormones and Behavior, 2016; (77): 167-181.

15. KALIL IR, AGUIAR AC. Silêncios nos discursos pró-aleitamento materno: uma análise na perspectiva de gênero. Estudos Feministas, Florianópolis, 2017; 25(2): 637-60.

16. KALIL IR, AGUIAR AC. Trabalho feminino, políticas familiares e discursos pró-aleitamento materno: avanços e desafios à equidade de gênero. Saúde Debate, 2016; 40(110): 208-223.

17. LABBOK MH. Sexualidade pós-parto e o método de amenorreia lactacional para contracepção. Clinical Obstetrics and Gynecology, 2015; 58(4): 915-927.

18. LANGER-GOULD A, et al. Amamentação, anos ovulatórios e risco de esclerose múltipla. Neurology, 2017, 89(6): 563569.

19. LIMA SP, et al. Percepção de mulheres quanto à prática do aleitamento materno: uma revisão integrativa. Cuidado é fundamental, 2019; 11(1): 248-254.

20. MINISTÉRIO DA SAÚDE. Saúde da criança: aleitamento materno e alimentação complementar. $2^{\mathrm{a}}$ edição Cadernos de Atenção Básica, no 23, Brasília - DF, Brasil, 2015. Disponível em: https://bvsms.saude.gov.br/bvs/publicacoes/saude_crianca_aleitamento_materno_cab23.pdf. Acessado em: 15 de maio de 2021.

21. MOIMAZ SAS, et al. Aleitamento materno: benefícios à saúde e implicações na saúde bucal. Revista Paulista de Odontologia, 2014; 36(4): 24-31.

22. MONTEIRO FR, et al. Influência da licença-maternidade sobre a amamentação exclusiva. Jornal de Pediatria, 2017; 93: 475-481.

23. MONTESCHIO CAC, et al. O enfermeiro frente ao desmame precoce na consulta de enfermagem à criança. Revista Brasileira de Enfermagem, 2015; 68(5): 869-875.

24. NUNES JT, et al. Qualidade da assistência pré-natal no Brasil: revisão de artigos publicados de 2005 a 2015 . Caderno de Saúde Coletiva, 2016; 24(2): 252-261.

25. OLIVEIRA DS, et al. Duração do aleitamento materno e fatores associados entre 1960 e 2000. Jornal de Pediatria, 2017; 93: 130-135.

26. PRADO CVC, et al. Early weaning from breastfeeding from mothers' perspective: a dialogical approach. Texto \& contexto Enfermagem, 2016; 25(2): 1-9.

27. ROLLINS NC, et al. Por que investir e o que será necessário para melhorar as práticas de amamentação? Epidemiologia e Serviços de Saúde, 2016; 25(1): 25-44.

28. SANTOS FS, et al. Breastfeeding and protection against diarrhea: an integrative review of literature. Einstein, 2015; 13(3): 435-40.

29. SANTOS GCP, et al. Os benefícios da amamentação para a saúde da mulher. Revista Saúde em Foco, 2017; 9: 225 228.

30. SANTOS ZB. Benefícios do Aleitamento Materno Exclusivo para o Lactente e para a Nutriz até o Sexto Mês. Revista Científica Multidisciplinar Núcleo do Conhecimento, 2018; 2: 84-109.

31. SILVA VA, et al. Aleitamento materno: indicadores e fatores associados a amamentação exclusiva num aglomerado urbano subnormal assistido pela Estratégia de Saúde da Família. Jornal de Pediatria, 2019; 95(3): 298-305.

32. SILVA CM, et al. Práticas educativas segundo os "Dez passos para o sucesso do aleitamento materno" em um Banco de Leite Humano. Ciência e Saúde coletiva, 2017; 22(5): 1661-1671.

33. SIPSMA HL, et al. Efeito da amamentação nos sintomas depressivos pós-parto entre mães adolescentes e adultas jovens. Journal of Maternal-Fetal and Neonatal Medicine, 2018; 31(11): 1442-1447.

34. VICTORA CG, et al. Amamentação no século 21: epidemiologia, mecanismos e efeitos ao longo da vida. Epidemiologia e Serviço em Saúde, 2016; 387: 1-24.

35. WIJDEN CVD, MANION C. Lactational amenorrhoea method for family planning. Cochrane Database Systematic Reviews, 2015; (10): 1-23. 